\title{
A study of reaction wheel configurations for a 3-axis satellite attitude control
}

\begin{abstract}
The satellite reaction wheel's configuration plays also an important role in providing the attitude control torques. Several configurations based on three or four reaction wheels are investigated in order to identify the most suitable orientation that consumes a minimum power. Such information in a coherent form is not summarized in any publication; and therefore, an extensive literature search is required to obtain these results. In addition, most of the available results are from different test conditions; hence, making them difficult for comparison purposes. In this work, the standard reaction wheel control and angular momentum unloading schemes are adopted for all the reaction wheel configurations. The schemes will be presented together with their governing equations, making them fully amenable to numerical treatments. Numerical simulations are then performed for all the possible reaction wheel configurations with respect to an identical reference mission. All the configurations are analyzed in terms of their torques, momentums and attitude control performances. Based on the simulations, the reaction wheel configuration that has a minimum total control torque level is identified, which also corresponds to the configuration with minimum power consumption.
\end{abstract}

Keyword: Reaction wheel; Satellite attitude control; Momentum management 